\title{
Valoración del recurso hídrico en el lavado del equipo T-90 Calima. Variables económicas y medio ambiente ${ }^{1}$
}

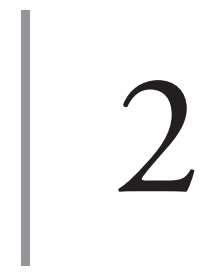

https://doi.org/10.21830/9789585380219.02

Nicolás Rodríguez Flórez"
Alexandra Ibargüen Asprilla

Resumen. Los recursos naturales son elementos esenciales de las políticas de gobierno para el desarrollo social y económico del país. Aunque Colombia es un territorio biodiverso, la continua sobreexplotación de los activos ambientales genera su escasez y, por lo tanto, pone en riesgo su disponibilidad para las generaciones futuras. Este capítulo analiza el recurso hídrico que se emplea en el lavado del equipo T-90 Calima de la Escuela Militar de Aviación (EMAVI) de acuerdo con los requerimientos técnicos y de seguridad establecidos en el Boletín FAC 1-1-691-L, así como los insumos, la maquinaria y demás recursos necesarios para este proceso. Se propone un método de investigación descriptivo, el cual parte de la caracterización de los elementos asociados al lavado del equipo T-90 Calima. Para ello, se realiza un análisis económico a fin de conocer los costos totales de la actividad y se realiza el análisis ambiental para medir la huella hídrica, que es indirecta y corresponde a las aguas residuales contaminadas con los insumos de limpieza, cuyos costos fueron calculados en el servicio de alcantarillado. Los resultados del estudio muestran que

1 El presente capítulo es resultado del proyecto de investigación titulado "Valoración del activo ambiental agua en el proceso de lavado del equipo T-90C en la Escuela Militar de Aviación Marco Fidel Suárez con respecto a las variables económica y medio ambiente". Los puntos de vista y los resultados presentados en este capítulo pertenecen a los autores y no reflejan necesariamente los de las instituciones participantes.

2 Oficial de la Fuerza Aérea Colombia. Administrador Aeronáutico y profesional en Ciencias Militares de la Escuela Militar de Aviación "Marco Fidel Suárez". Piloto de RpA. Comandante Elemento de Ataque Escuadrón de RPA del Grupo de Combate N. 51 del Comando Aéreo del Combate No. 5 (CACOM-5). Orcid: https://orcid.org/9189-0148-0003-0000 - Contacto: nicolas.rodriguezf@fac.mil.co

3 Estudiante de la Maestría en Administración. Administradora de empresas y tecnóloga en Gestión Ejecutiva de la Universidad del Valle. Se ha desempeñado en cargos de gestión administrativa y financiera en propiedad horizontal. Orientadora de Defensa en el Programa de Administración Aeronáutica de la Escuela Militar de Aviación "Marco Fidel Suárez", Cali, Colombia. Coordinadora del Semillero de Investigación Siloga y directora de trabajos de grado en el área de Gestión Ambiental. OrCID: https://orcid.org/0000-0001-8117-3154 - Contacto: alexandra.ibarguen@emavi.edu.co 
el consumo de agua potable empleado es de 9.576 litros, que corresponden al lavado de las 24 aeronaves que componen la flota del equipo T-90 Calima, y el costo del proceso es $\$ 4$ 4229.675. Por lo tanto, como conclusión se recomienda emplear fuentes alternativas, como agua de pozo, agua de lluvias y aguas grises, para optimizar el uso del recurso hídrico y utilizar insumos de menor impacto con el medio ambiente.

Palabras clave: activos ambientales; agua; desarrollo sostenible; Escuela Militar de Aviación; impacto ambiental.

\section{Introducción}

Los recursos naturales son elementos esenciales para el desarrollo social y económico de un país, de manera que su cuidado, protección y conservación es responsabilidad de toda la ciudadanía, el Estado, la universidad y la empresa pública y privada.

La Ley 99 de 1993, la cual crea el Sistema Nacional Ambiental y el Ministerio de Medio Ambiente, expresa en su artículo 103:

Del apoyo de las Fuerzas Armadas. Las Fuerzas Armadas velarán en todo el territorio nacional por la protección y defensa del medio ambiente y los recursos naturales renovables y por el cumplimiento de las normas dictadas con el fin de proteger el patrimonio natural de la Nación, como elemento integrante de la soberanía nacional.

Sin embargo, cabe tener en cuenta que estas instituciones del Estado realizan actividades en las que se utilizan activos ambientales. Este es el caso en la Escuela Militar de Aviación (EMAvi) con el lavado de las aeronaves, proceso en el que se hace uso de los recursos naturales con la intención de prestar un servicio y dar cumplimiento a la misión de "volar, entrenar y combatir para vencer y dominar en el aire, el espacio y ciberespacio, en defensa de la soberanía, la independencia, la integridad territorial, el orden constitucional y contribuir a los fines del Estado" (Fuerza Aérea Colombiana, 2019).

Actualmente, la EMAVI cuenta con aeronaves de instrucción, entre las cuales se encuentra el equipo T-90 Calima, aeronave diseñada y manufacturada por la Corporación de la Industria Aeronáutica Colombiana (CiAT), con fuselaje en fibra de vidrio y carbono, y con capacidad para dos tripulantes. De 
acuerdo con los requerimientos técnicos, las aeronaves deben tener un proceso de lavado mensual estipulado en el Boletín FAC 1-1-691-L, pero no existe una valoración económica y ambiental de los costos asociados a esta actividad. Por este motivo surge el siguiente interrogante: ¿Cómo evaluar el recurso hídrico en el proceso de lavado de la aeronave T-90 Calima?

\section{Metodología}

Se realizó un estudio descriptivo del proceso de lavado de una aeronave T-90 Calima, a fin de obtener información que permitió analizar las actividades de acuerdo con las variables recurso económico y medio ambiente planteadas en la investigación. Cabe recordar que los estudios descriptivos miden, evalúan o recolectan datos sobre diversos conceptos (variables), aspectos, dimensiones o componentes del fenómeno que se investiga (Hernández-Sampieri et al., 2006, p. 102).

Para realizar la evaluación del recurso hídrico en el proceso de lavado del equipo T-90 Calima se partió de la documentación técnica establecida en el Boletín FAC 1-1-691-L del Grupo Técnico de la EMAVı para conocer la regularidad de la actividad. A partir de esta información se realizó la caracterización de los elementos asociados al proceso de lavado.

Asimismo, partiendo de los costos económicos generados por el recurso hídrico, se estableció la huella hídrica asociada a las aguas residuales que resultan del proceso de lavado del equipo de instrucción T-90 Calima. Finalmente, se presentan recomendaciones de fuentes alternativas para optimizar el uso del recurso hídrico y de insumos de menor impacto con el medio ambiente.

La evaluación fue aplicada a la flota de 24 aeronaves T-90 Calima, que representa el 70,58 \% de las aeronaves de instrucción con las que cuenta la EMAVI.

\section{Marco conceptual}

El concepto técnico de "recursos naturales no renovables" proviene de la ecología y de la economía. Se pueden definir los recursos naturales como aque- 
llos elementos de la naturaleza y del medio ambiente. A su vez, los recursos naturales se clasifican en renovables y no renovables. Los primeros son aquellos que la propia naturaleza repone periódicamente mediante procesos biológicos o de otro tipo, esto es, que se renuevan por sí mismos. Por el contrario, los recursos no renovables se caracterizan por cuanto existen en cantidades limitadas y no están sujetos a una renovación periódica por procesos naturales (Sentencia C-221, 1997).

Los activos ambientales son definidos como territorios o espacios físicos que sustentan un determinado tipo de ecosistema y que ofrecen bienes y servicios ambientales (Anzar \& Estruch, 2015). Tanto los bienes como los servicios ambientales son suministrados por la naturaleza, pero existen distinciones de uso, valoración y aprovechamiento.

Desafortunadamente, la mayoría de los activos ambientales sufren en la actualidad una agresión importante que está provocando su paulatina degradación (Anzar \& Estruch, 2015, p. 12). El recurso hídrico, como activo ambiental, es indispensable para la vida y el desarrollo económico y social, de manera que ante la creciente demanda resulta vital valorarlo en los procesos productivos de una nación, población u organización.

Según Francisco Correa Restrepo, economista y especialista en evaluación socioeconómica de proyectos de la Universidad de Antioquia, el uso descontrolado de los activos ambientales y el impacto que esto tiene en la economía y el medio ambiente inicialmente empeora la calidad ambiental, debido a la creciente necesidad de productos y servicios de la sociedad que demanda un mayor uso de los recursos naturales (Correa, 2004, pp. 93-94), por tanto, se establece que mientras exista capacidad económica, la sociedad no prestará la suficiente atención al alto consumo de los activos ambientales como el agua.

El agua es un elemento esencial para la vida que contribuye a satisfacer las necesidades de los seres humanos, sin embargo, la explotación excesiva y la interrupción del ciclo del agua causan la escasez del recurso natural. Por ello, la definición tradicional del agua - "líquido transparente, incoloro, inodoro e insípido en estado puro, cuyas moléculas están formadas por dos átomos de hidrógeno y uno de oxígeno, y que constituye el componente más abundante de la superficie terrestre y el mayoritario de todos los organismos vivos" (Real Academia Española 
de la Lengua, 2014)_ - se ha ido transformando por la economía globalizada de un bien común a uno económico en crisis global.

La problemática del agua constituye una crisis ecológica con causas comerciales, pero sin soluciones de mercado (Shiva, 2003, p. 32), puesto que desde el ámbito comercial se impulsaron políticas de mercantilización y privatización de los servicios de abastecimiento y saneamiento del agua, pero no se establecieron medidas para la protección y el uso racional.

Ante la preocupación imperante por la crisis global del agua, en la Conferencia Internacional sobre el Agua y el Medio Ambiente (CIAMA), celebrada en Dublín, Irlanda, del 26 al 31 de enero de 1992, se formuló la Declaración de Dublín sobre el Agua y el Desarrollo Sostenible, cuyo cuarto principio establece: "El agua tiene un valor económico en todos sus diversos usos en competencia a los que se destinan y debería reconocérsele como un bien económico (Organización de las Naciones Unidas para la Educación, la Ciencia y la Cultura [UNESCO], 1992).

Según el Diccionario de la Real Academia Española de la Lengua, el acto de valorar supone reconocer, estimar o apreciar el valor o mérito de alguien o algo. Asimismo, el concepto de valor se entiende como el grado de utilidad o aptitud de las cosas para satisfacer las necesidades o proporcionar bienestar o deleite.

Por su parte, en su libro Esquemas de historia del pensamiento económico, Sanz (2006) afirma que la teoría de valor es una de las claves del pensamiento económico. Cabe precisar que el concepto de valor ha tenido dos significados diferentes en la historia del pensamiento económico:

1. Valor de uso: utilidad proporcionada por un bien o servicio.

2. Valor de cambio: cantidad de otro bien que se puede conseguir a cambio (Sanz, 2006, p. 287).

Precisamente, la valoración económica de los activos ambientales puede contribuir a fomentar su cuidado y protección. Según la Guía de aplicación de la valoración económica ambiental, del Ministerio del Medio Ambiente y Desarrollo Sostenible, la valoración económica ambiental consiste en la "asignación de valores cuantitativos a los bienes y servicios proporcionados por los recursos naturales, independientemente de si existen o no precios de mercado que ayuden a hacerlo" (Ministerio del Medio Ambiente y Desarrollo Sostenible, 
2015, p. 15). Por lo tanto, valorar un activo ambiental permite establecer la importancia de los bienes y servicios que proporciona la naturaleza.

$\mathrm{Al}$ respecto, en su artículo "Valoración económica ambiental: el problema del costo social", Raffo (2015) argumenta que la valoración ambiental puede definirse formalmente como un "conjunto de técnicas y métodos que permiten medir las expectativas de beneficios y costos derivadas de algunas de las acciones como: uso de un activo ambiental, realización de una mejora ambiental y generación de un daño ambiental" (p. 108). En este sentido, la valoración del agua resulta vital para todos los actores involucrados.

Para las Naciones Unidas, "el agua está en el epicentro del desarrollo sostenible y es fundamental para el desarrollo socioeconómico, la energía y la producción de alimentos, los ecosistemas saludables y para la supervivencia misma de los seres humanos" (Organización de las Naciones Unidas [ONU], 2019), puesto que conocen las problemáticas del agua como sustancia fundamental para la vida e indispensable para todas las actividades humanas.

Precisamente, la Asamblea General de las Naciones Unidas adoptó en septiembre de 2015 la Agenda 2030 para el Desarrollo Sostenible, la cual plantea diecisiete Objetivos de Desarrollo Sostenible (ODs). Entre estos, el sexto establece "garantizar la disponibilidad de agua y su gestión sostenible y el saneamiento para todos". Las metas de este objetivo cubren tanto los aspectos del ciclo del agua como los sistemas de saneamiento, y se espera que la consecución de este objetivo contribuya en el progreso de otros ODs, principalmente en salud, educación, crecimiento económico y medio ambiente (ONU, 2019).

Ante este escenario, en todo el mundo existe la necesidad de crear estrategias para el cuidado, la protección y la conservación de los recursos naturales. Sin embargo, debido a que el agua es un activo ambiental de alta demanda por los requerimientos humanos y los propios de los procesos industriales, el uso irracional afecta la calidad y la disponibilidad del recurso. Es por ello que el concepto de desarrollo sostenible es inherente a los recursos naturales y los activos ambientales.

La primera definición clásica del concepto de desarrollo sostenible se presentó en el Informe Brundtland: "Desarrollo sostenible que satisface las necesidades del presente sin comprometer la capacidad de las generaciones 
futuras". De acuerdo con Gómez (2020), a partir de esta conceptualización inicial, Sérgio Buaquer definió el desarrollo sostenible como "un proceso cualitativo y cuantitativo de cambio social que compatibiliza, en el tiempo y en el espacio, el crecimiento económico, la conservación ambiental y la equidad social" (p. 27).

Teniendo en cuenta la anterior definición, se puede afirmar que el desarrollo sostenible integra las dimensiones ecológica, económica y social. En este sentido, las organizaciones que buscan establecer políticas y acciones para lograr un crecimiento económico deberán respetar el medio ambiente y ser socialmente equitativas para alcanzar el crecimiento económico (Artazan, 2002). Así, las diferentes dimensiones del desarrollo sostenible buscan la interacción entre los distintos elementos que lo componen para transformar, fomentar y ofrecer opciones racionales y eficientes de los recursos naturales.

\section{Resultados}

\section{Caracterización de los elementos asociados al proceso de lavado del equipo T-90 Calima}

Después de observar el proceso de lavado y revisión del Boletín FAC 1-1-691-L, se estableció que se requiere limpieza por lo menos una vez al mes para eliminar agentes corrosivos que deterioren el fuselaje de las aeronaves. Los elementos asociados al proceso se describen en la tabla 1.

Tabla 1. Elementos asociados al proceso de lavado del equipo T-90 Calima

\begin{tabular}{ll}
\hline \multicolumn{1}{c}{ Elemento } & \multicolumn{1}{c}{ Características } \\
\hline Personal & Proceso manual, equipo de protección \\
Maquinaria & Hidrolavadora Kärcher HDS 3.5/23-4 Eg \\
& $\begin{array}{l}\text { Agua potable, shampoo MIL-PRF-8557 (CCA), } \\
\text { alcohol isopropílico TTI-735, nafta alifático } \\
\text { Insumos }\end{array}$ \\
& TT-N-95 \\
\hline
\end{tabular}

Fuente: Elaboración propia. 
Asimismo, se establecieron las actividades del proceso de lavado de una aeronave de la flota T-90 Calima y el tiempo total requerido (70 minutos), los cuales se discriminan en la tabla 2.

Tabla 2. Actividades y tiempos de ejecución

\begin{tabular}{lc}
\hline \multicolumn{1}{c}{ Actividad } & Tiempo de ejecución \\
\hline Aplicación del detergente con agua & 20 minutos \\
Actuación del detergente en la aeronave & 20 minutos \\
Limpieza del detergente con agua a presión & 10 minutos \\
Secado & 20 minutos \\
\hline Total & $\mathbf{7 0}$ minutos \\
\hline
\end{tabular}

Fuente: Elaboración propia.

\section{Análisis económico de los costos asociados al proceso de lavado del equipo T-90 Calima}

A continuación, la tabla 3 presenta la discriminación de cada uno de los costos económicos asociados al proceso de lavado del equipo T-90 Calima. En primer lugar, los costos de personal (tabla 3) corresponden a un funcionario FAC de grado AS16. Asistencial.

Tabla 3. Costos de personal

\begin{tabular}{lr}
\hline \multicolumn{1}{c}{ Ítem } & \multicolumn{1}{c}{ Valores } \\
\hline Salario (incluyendo primas) & $\$ 2{ }^{\prime} 011.113$ \\
Seguridad social & $\$ 321.094$ \\
\hline Total & $\$ 2 ’ 332.208$ \\
\hline
\end{tabular}

Fuente: Elaboración propia.

En segundo lugar, la tabla 4 muestra los costos por concepto de acueducto y alcantarillado, teniendo en cuenta el caudal de la maquina Kärcher y el tiempo en el que se utiliza el equipo durante el lavado del equipo T-90 Calima. 
Los costos fueron calculados de acuerdo con la tarifa oficial establecida por la empresa prestadora del servicio: Empresas Municipales de Cali (EMCALI).

Tabla 4. Costos del consumo de acueducto y alcantarillado

\begin{tabular}{lrrrrr}
\hline \multicolumn{1}{c}{ Ítem } & Tarifa m3 & \multicolumn{1}{c}{ Tarifa $\mathbf{x}$ litro } & Tiempo uso & Cantidad & \multicolumn{1}{c}{ Costo } \\
\hline Acueducto & $\$ 2.278$ & $\$ 2.28$ & 30 minutos & 399 litros & $\$ 909,72$ \\
Alcantarillado & $\$ 2.592$ & $\$ 2.59$ & 30 minutos & 399 litros & $\$ 1.033,41$ \\
\hline Total & $\mathbf{\$ 4 . 8 7 0}$ & $\mathbf{\$ 5}$ & $\mathbf{6 0}$ minutos & $\mathbf{7 9 8}$ litros & $\mathbf{\$ 1 . 9 4 3 , 1 3}$ \\
\hline
\end{tabular}

Fuente: Elaboración propia.

En tercer lugar, la tabla 5 discrimina los insumos de limpieza y cantidades requeridas para lavar una aeronave de la flota T-90 Calima.

Tabla 5. Costos asociados a los insumos de limpieza

\begin{tabular}{lrrc}
\hline \multicolumn{1}{c}{ Elemento } & \multicolumn{1}{c}{ Costo } & Cantidad utilizada & Costo por lavado \\
\hline Shampoo MIL-PRF-8557 & $\$ 295.500 / 5 \mathrm{gal}$ & $0,66 \mathrm{gal}=0,25 \mathrm{~L}$ & $\$ 19.532$ \\
Alcohol isopropílico TTI-735 & $\$ 30.000 \mathrm{~L}$ & $0,5 \mathrm{~L}$ & $\$ 15.000$ \\
TT-N-95 nafta alifática & $\$ 237.831 \mathrm{~L}$ & $0,5 \mathrm{~L}$ & $\$ 118.915$ \\
Diésel & $\$ 8.151 \mathrm{~L}$ & $6,6 \mathrm{~L}$ & $\$ 53.796$ \\
\hline Total & & & $\$ \mathbf{2 0 7 . 2 4 3}$ \\
\hline
\end{tabular}

Fuente: Elaboración propia.

Finalmente, en la tabla 6 se integran los costos totales asociados al proceso de lavado de la flota de 24 aeronaves del equipo T-90 Calima.

Tabla 6. Costos totales del proceso de lavado del equipo T-90 Calima

\begin{tabular}{lrrrr}
\hline \multicolumn{1}{c}{ Ítem } & Costo mensual & Costo por hora & Costo lavado & Costo flota T-90C \\
\hline Personal & $\$ 2$ '332.208 & $\$ 9.718$ & $\$ 11.337$ & $\$ 272.090$ \\
Maquinaria & $\$ 53.796$ & $\$ 8.150$ & $\$ 9.509$ & $\$ 228.225$ \\
Insumos & $\$ 153.447$ & $\$ 153.447$ & $\$ 153.447$ & $\$ 3.682 .728$ \\
Agua & $\$ 46.632$ & $\$ 3.886$ & $\$ 1.943$ & $\$ 46.632$ \\
\hline Total & $\mathbf{\$ 2} \mathbf{5 8 6 . 0 8 3}$ & $\mathbf{\$ 1 7 5 . 2 0 1}$ & $\mathbf{\$ 1 7 6 . 2 3 6}$ & $\mathbf{\$ 4 2 2 9 . 6 7 5}$ \\
\hline
\end{tabular}

Fuente: Elaboración propia. 


\section{Análisis de la huella hídrica asociada al proceso de lavado del equipo T-90 Calima}

La huella hídrica como herramienta de gestión amplía las expectativas en cuanto al análisis y estudio de los recursos hídricos y su entorno, así como la identificación de los agentes de presión que en ellos intervienen. También como herramienta de planeación del manejo del recurso hídrico y finalmente para generar conciencia sobre el impacto de las actividades diarias en la degradación de los recursos naturales.

Teniendo en cuenta el consumo de agua potable requerido en el proceso de lavado del equipo T-90 Calima, se identificó que la huella hídrica es indirecta y que corresponde a las aguas residuales contaminadas con los insumos de limpieza. Adicionalmente, existen unos costos por el vertimiento de las aguas residuales, los cuales también fueron calculados con la tarifa oficial establecida por la empresa prestadora del servicio, EmCALI, en el concepto de alcantarillado.

\section{Discusión}

A continuación se discuten los principales resultados de la investigación. En primer lugar, el consumo de agua potable en el proceso de lavado del equipo objeto de estudio es de 399 litros, teniendo en cuenta que la actividad de limpieza se realice una vez al mes, de acuerdo con el Boletín FAC 1-1-691-L. Sin embargo, existen factores ambientales que pueden hacer más recurrente los procesos de limpieza y, por consiguiente, se eleva la demanda de uso del activo ambiental.

En segundo lugar, se debe tener en cuenta que el consumo de agua potable se estableció para la aeronave de instrucción T-90 Calima, de dimensiones: 266 inch - 6,75 m, ancho: 344 inch - 8,73 m y altura: 96 inch - 2,43 m, categorizado como avión pequeño, de manera que si se realiza el análisis en una aeronave de mayor tamaño los costos y el uso del activo ambiental se incrementan.

En tercer lugar, al observar los costos del consumo de agua potable se evidencia que el servicio de alcantarillado es más costoso por el estimativo de la disposición de aguas residuales, de acuerdo con la Resolución CRA 800 de julio 
28 de 2017 de la Comisión de Regulación de Agua Potable y Saneamiento Básico, por la cual se instaura la opción de medición de vertimientos en el servicio público domiciliario de alcantarillado. Por lo tanto, se deben contemplar fuentes alternativas de agua para realizar esta actividad para disminuir los costos por servicio de acueducto y alcantarillado.

En cuarto lugar, en la Resolución CRA 2718 de 2003 de la Comisión de Regulación de Agua Potable y Saneamiento Básico se define la demanda del servicio de alcantarillado como la "equivalente a la demanda del servicio de acueducto, más el estimativo de la disposición de aguas residuales de aquellos usuarios que posean fuentes alternas o adicionales de abastecimiento de agua que viertan al alcantarillado". Sin embargo, la Comisión de Regulación de Agua Potable y Saneamiento Básico estableció en su marco normativo que los usuarios pueden solicitar la medición de los vertimientos, teniendo en cuenta que pueden existir diferencias en la correlación entre los consumos de acueducto y alcantarillado, siendo esta una opción para disminuir los costos facturados.

En quinto lugar, se debe señalar que actualmente hay poblaciones del país sin acceso al servicio de agua potable y saneamiento básico (La Guajira), y que las enfermedades asociadas al consumo de agua sin tratamiento proveniente de pozos y ríos han desencadenado consecuencias lamentables. Por eso contrasta que se utilicen cientos de litros de agua potable para el lavado de aeronaves.

En sexto lugar, de acuerdo con la Organización Mundial de la Salud (OMs), una persona debe consumir en promedio cien litros de agua para satisfacer las necesidades tanto de consumo como de higiene, siendo el agua cada vez más escasa en algunas regiones del país y del mundo. Por lo tanto, reducir el consumo de agua potable en el proceso de lavado de aeronaves posibilita el acceso al recurso a poblaciones vulnerables, a costos razonables, puesto que la limitación encarece el valor.

En séptimo lugar, cabe mencionar que entidades públicas y privadas están en la búsqueda constante de disminuir los costos de operación, y la Fuerza Aérea Colombiana no es ajena a este panorama. El uso de fuentes alternativas de agua, como agua de pozo, aguas lluvias y aguas grises, reduce los costos 
económicos por el servicio de acueducto y alcantarillado, así como el impacto ambiental en el desarrollo de esta actividad. Sin embargo, estas alternativas requieren mayores estudios para determinar los posibles agentes.

En octavo lugar, se debe tener en cuenta que el uso de aguas lluvias y aguas grises requiere estudios más profundos antes de implementarlo, puesto que este tipo de agua puede contener agentes que afecten el fuselaje de las aeronaves.

En noveno lugar, el sector aeronáutico ha desarrollado técnicas de lavado en seco para reducir el consumo de agua potable, pero se deben analizar los costos de los insumos, la efectividad, el tiempo y el recurso humano requerido en el proceso de limpieza.

Finalmente, se debe tener presente que en el mercado de productos de limpieza se han desarrollado insumo cien por ciento naturales, aprobados y certificados para uso en aeronaves civiles y militares, los cuales podrían ser un referente de procesos amigables y sostenibles con el medio ambiente.

\section{Conclusiones}

El consumo de agua potable para el lavado de una aeronave del equipo T-90 Calima es de 399 litros, de manera que el lavado de la flota completa implica el uso de 9.576 litros. Si este consumo se dimensiona anualmente, sería de 114.912 litros, si se realiza un solo lavado mensual, pero se debe tener en cuenta que los requerimientos de limpieza pueden variar por agentes en el ambiente o por las condiciones climáticas, lo cual podría elevar considerablemente el consumo del activo ambiental en esta actividad.

Los costos económicos mensuales totales de lavar una vez al mes las 24 aeronaves que componen la flota T-90 Calima es de \$4229.675. Ante este panorama, vale la pena considerar que existen innovaciones en el sector aeronáutico, como la técnica de lavado en seco, que se podrían implementar para disminuir el impacto por el uso de agua potable y, a su vez, reducir los costos de operación.

Finalmente, se concluye que, al dimensionar el consumo de agua potable que se utiliza para el lavado de todas las aeronaves adscritas a la Fuerza Aérea 
Colombiana, la cantidad y los costos resultan onerosos para la institución, adicional al impacto ambiental por el consumo del líquido, que en contraste escasea en algunas poblaciones o regiones del país.

\section{Referencias}

Anzar, J., \& Estruch, A. (2015). Valoración de activos ambientales: Teoría y casos (2.a ed.). Editorial Universidad Politécnica de Valencia. https://riunet.upv.es/bitstream/ handle/10251/66822/PDF-Aznar\%3BEstruch\%20-\%20VALORACI\%C3\%93N\%20 DE\%20ACTIVOS\%20AMBIENTALES.pdf?sequence=1

Artazan, M. (2002). Teoría de las tres dimensiones de desarrollo sostenible. Ecosistemas, 11(2). https://www.revistaecosistemas.net/index.php/ecosistemas/article/view/614

Correa, F. (2004). Crecimiento económico y medio ambiente: Una revisión analítica de la hipótesis de la curva ambiental de Kuznets. Semestre Económico, 7(14), 74-104. https:// revistas.udem.edu.co/index.php/economico/article/view/1131

Fuerza Aérea Colombiana. (2019). Estrategia para el desarrollo aéreo y espacial de la Fuerza Aérea Colombia. Fuerza Aérea Colombiana.

Gómez, I. (2020). Desarrollo sostenible. Editorial Elearning S. L.

Hernández-Sampieri, R., Fernández-Collado, C., \& Baptista Lucio, P. (2006). Metodología de la investigación (4.a ed.). McGraw Hill.

Ministerio de Medio Ambiente y Desarrollo Sostenible. (2015). Guía de aplicación de la valoración económico ambiental. https://www.minambiente.gov.co/images/NegociosVerdesysostenible/ pdf/valoracion_economica_ambiental/Gu\%C3\%ADa_de_aplicaci\%C3\%B3n_de_la_ VEA_Comprimida.pdf

Organización de las Naciones Unidas [ONU]. (2019). Desafíos globales: Agua. https://www. un.org/es/global-issues/water

Organización de las Naciones Unidas para la Educación, la Ciencia y la Cultura [UNESCO]. (1992). Declaración de Dublín sobre el agua y el desarrollo sostenible. https://es.unesco. org/news/nuestra-agua-nuestro-mundo-conferencia-internacional-del-agua-unesco-pide-cambio-paradigma

Raffo, E. (2015). Valoración económica ambiental: El problema del costo social. Industrial Data, 18(1), 108-118. https://doi.org/10.15381/idata.v18i1.12073

Real Academia Española de la Lengua. (2014). Valorar. En Diccionario de la Lengua Española. https://dle.rae.es/valorar

Sanz Serrano, J. (2006). Esquemas de historias del pensamiento económico. Editorial Universidad de Sevilla.

Sentencia C-221. (1997). Corte Constitucional. Magistrado ponente: Alejandro Martínez Caballero. https://www.corteconstitucional.gov.co/relatoria/1997/C-221-97.htm

Shiva, V. (2003). La guerra del agua: Privatización, contaminación y lucro. Siglo XXI Editores. 